\title{
Melatonin Enhances Chondrocytes Survival Through Hyaluronic Acid Production and Mitochondria Protection
}

\section{Type}

Research paper

\section{Keywords}

melatonin, mitochondrion, hyaluronic acid, NF-KB, chondrocyte

\begin{abstract}
Introduction

Melatonin, an indoleamine synthesized mainly in the pineal gland, plays a key role in regulating the homeostasis of bone and cartilage. Reactive oxygen species (ROS) are released during osteoarthritis $(\mathrm{OA})$ and associated with cartilage degradation. Melatonin is a potent scavenger of ROS.
\end{abstract}

\section{Material and methods}

We used rat chondrocytes as a research objective in our study and investigated the effect of melatonin. We evaluated cell viability by MTS, cell apoptosis and mitochondrial transmembrane potential (MMP) by flow cytometry, hyaluronic acid (HA) concentrations by ELISA assay, relative gene and protein expression by RT-qPCR and WB assay, the subcellular location of nuclear factor KB (NF-KB) p65 and Rh-123 dye by immunofluorescence, oxygen consumption rate (OCR) by Seahorse equipment.

\section{Results}

MTS and flow cytometry assay indicated that melatonin prevented $\mathrm{H} 2 \mathrm{O} 2$-induced cell death. Melatonin suppressed the expression of HAS1 both from mRNA and protein level in time- and dosedependent manners. Furthermore, melatonin treatment attenuated the decrease in HA production in response to $\mathrm{H} 2 \mathrm{O} 2$ stimulation and repressed the expression of cyclooxygenase-2 (COX-2).

Melatonin significantly decreased the nuclear volume of p65 and inhibited the expression of p50 induced by $\mathrm{H} 2 \mathrm{O} 2$. In addition, melatonin restored the MMP and OCR of mitochondria, exerted mitochondrial protection function.

\section{Conclusions}

Melatonin has a protective effect on chondrocytes, possibly by upregulating HA synthesis and inhibiting the NF-kB signaling. 


\section{Melatonin Enhances Chondrocytes Survival Through Hyaluronic Acid Production and Mitochondria Protection}

Jingjing Wang ${ }^{1}$, Amrish Thapa ${ }^{2}$, Ying Xie ${ }^{1}$, Jing Guo ${ }^{1}$, Xin $\mathrm{Li}^{1}$, Hongmei Jiang ${ }^{1}$, Yixuan Wang ${ }^{1}$, Ziyi Peng ${ }^{1}$, Sheng Wang ${ }^{1}$, Jingya Wang ${ }^{1}$, Xianhui $\mathrm{Li}^{3}$, Lingqun $\mathrm{Ye}^{4}$, Zhiqiang Liu $^{1}$

${ }^{1}$ Tianjin Key Laboratory of Cellular Homeostasis and Human Diseases, School of Basic Medical Science, Tianjin Medical University; Department of Physiology and Pathophysiology, School of Basic Medical Science, Tianjin Medical University, Heping, Tianjin, 300070 China

${ }^{2}$ Department of Cardiology, Tianjin First Central Hospital, Tianjin, 300192 China

${ }^{3}$ Department of health services, Logistics University of People's Armed Police Force, Tianjin, 300039 China

${ }^{4}$ Center for Translational Research in Hematological Malignancies, Cancer Center, Houston Methodist Hospital, Houston, TX, 77030, USA

\section{Correspondence:}

Zhiqiang Liu, M.D., Ph.D., Professor, Tianjin Key Laboratory of Cellular Homeostasis and Human Diseases, School of Basic Medical Science, Tianjin Medical University; Department of Physiology and Pathophysiology, School of Basic Medical Sciences, Heping, Tianjin, 300070 China; Email: zhiqiangliu@tmu.edu.cn 


\begin{abstract}
Melatonin, an indoleamine synthesized mainly in the pineal gland, plays a key role in regulating the homeostasis of bone and cartilage. Reactive oxygen species (ROS) are released during osteoarthritis (OA) and associated with cartilage degradation. Melatonin is a potent scavenger of ROS. Herein, we investigated the effect of melatonin on rat chondrocytes. Melatonin prevented $\mathrm{H}_{2} \mathrm{O}_{2}$-induced cell death. RNA-sequencing identifies hyaluronan synthase 1 (HAS1) as one of the most

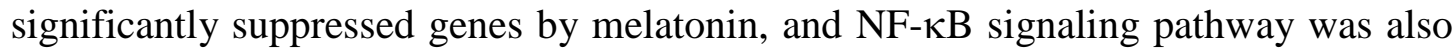
suppressed as well. Furthermore, melatonin treatment attenuated the decrease in hyaluronic acid (HA) production in response to $\mathrm{H}_{2} \mathrm{O}_{2}$ stimulation and repressed the expression of cyclooxygenase-2 (COX-2). In addition, melatonin protects chondrocytes from $\mathrm{H}_{2} \mathrm{O}_{2}$ induced mitochondrial damage. Taken together, our results suggest that melatonin has a protective effect on chondrocytes, possibly by upregulating HA synthesis and inhibiting the NF- $\mathrm{KB}$ signaling.
\end{abstract}

Keywords: melatonin; chondrocyte; hyaluronic acid; mitochondrion; NF- $\mathrm{KB}$ 


\section{Introduction}

Osteoarthritis (OA), a chronic degenerative arthritis, is the most popular joint disease in the elderly. The estimated prevalence of OA among people aged 50-59 years is 35\% and $55 \%$ for people older than 70 years $[1,2]$. OA is characterized by progressive damage of articular cartilage, breaking down of hyaluronic acid (HA) and underlying bone, causing pain, joint stiffness, and functional limitation. The causes and mechanisms of osteoarthritis remain to be fully understood. It is widely considered that inflammation is a feature of OA. Therefore, it is critical to understand the mechanisms underlying the inflammation of OA in order to develop more effective therapies.

Hyaluronic Acid Synthases (HASs) are transmembrane synthases responsible for HA generation and transportation through the plasma membrane to the pericellular matrix. Depending on the type of HAS, HA is generated with different length[3]. In addition, joints of OA patients show more aggressive histological pattern, characterized by chondrocyte hypertrophy, apoptosis as well as synoviocyte hyperplasia[4]. Nitric oxide (NO), superoxide, and the product of their reaction, peroxynitrite play fundamental roles in $\mathrm{OA}$ and induces apoptosis and necrosis in chondrocyte[5]. Excessive reactive oxygen species (ROS) during the inflammation of the synovial membranes contributes to chondrocytes and synoviocytes injury, senescence, transformation[6] and is also associated with cartilage degradation. The nuclear factor- $\kappa \mathrm{B}(\mathrm{NF}-\kappa \mathrm{B})$ signaling plays an essential role in inflammation[7], and it has two kinds of activation patterns which are named canonical and non-canonical NF- $\mathrm{B}$ signaling pathways[8]. Transcription factor family of NF- $\kappa \mathrm{B}$ signaling consists $\mathrm{p} 50$, p52, p65 (RelA), cRel, RelB, and its activity is tightly regulated by interaction with inhibitory I $\mathrm{KB}$ proteins[9]. The canonical pathway induced by phosphorylation and ubiquitin-dependent degradation of either $\mathrm{I} \kappa \mathrm{B} \alpha$ or $\mathrm{I} \kappa \mathrm{B} \beta$ and consequent the nuclear translocation of the p50 protein bound to either c-Rel or p65[10]. The NF- $\kappa B$ pathway is widely involved in OA pathophysiology through various effects[11]. Recently, numerous evidences have indicated that NF- $\kappa \mathrm{B}$ is activated by moderate ROS levels 
by different mechanisms[12]. Nevertheless, knowledge about mitigating the ROS-mediated inflammation drug OA remains not fully understood.

In joint of OA, synoviocytes transform into an aggressive and invasive fibroblast-like phenotype, break down cartilage by the action of matrix metalloproteinases[13]. Chondrocytes respond to the accumulation of biochemical and inflammatory insults by shifting into a degradative and hypertrophy-like status, accompanied by aberrant matrix production and elevated aggrecanase and collagenase activities[14]. Chondrocyte hypertrophy is catastrophic and could trigger a cascade of events, eventually causing permanent cartilage damage[15]. Notably, the cartilage is usually hypocellular in late-stage OA, often accompanied by lacunar emptying and osteoarthritic cartilage apoptosis[5]. Understanding of the mechanism involved in chondrocyte function and HA regulation is of great value to cure OA in the future.

Melatonin (N-acetyl-5-methoxytryptamine) is an indoleamine secreted primarily in the pineal gland under the rhythmical control of the suprachiasmatic nucleus (SCN) and the light/dark cycle[16]. Convincing evidence has demonstrated that melatonin regulates a variety of physiological functions, such as sleep-wake cycle, circadian rhythms, reproduction, aging, and neuroendocrine actions[17]. Notably, melatonin exerts anti-inflammatory effects through various pathways, such as modulating $\mathrm{NF}-\kappa \mathrm{B}$ pathway or cytokine secretion[18]. Melatonin can also provide antioxidant protection by scavenging excessive free radicals and increasing the synthesis of intracellular antioxidant enzymes. Notably, melatonin supplementation has shown its effectiveness in preventing the progression of osteoporosis and OA. However, the mechanisms remain unclear.

In this study, we aim to investigate the effects of melatonin on HA production and chondrocytes survival under oxidative stress and investigate the underlying mechanism. Our study might shed light on the development of novel therapeutic strategies for OA. 


\section{Materials and Methods}

\section{Cell isolation and culture}

All experiments were approved by the Animal Care and Use Committee of Tianjin Medical University. Chondrocytes were isolated from slices of the knee and hip-joint cartilage of 6-week-old male rat. After collected by centrifugation, chondrocytes were resuspended and seeded in Dulbecco's Modified Eagle Medium (DMEM, Gibico, USA), supplemented with 10\% fetal bovine serum (FBS, Hyclone, USA), $10 \%$ $\mathrm{NaHCO}_{3}, 100$ Units $/ \mathrm{mL}$ penicillin plus $100 \mu \mathrm{g} / \mathrm{ml}$ streptomycin at $37^{\circ} \mathrm{C}$ in humidified $5 \% \mathrm{CO}_{2}$ as monolayer culture.

\section{Enzyme-linked immunosorbent assay (ELISA)}

Chondrocytes were seeded in a 12 -well plate at a density of $10^{5}$ cells/well, then treated with $1 \mu \mathrm{M}$ melatonin (Sigma-Aldrich Corp., MO, USA) for different periods $(0,3,6,12,24,48 \mathrm{hr})$ or treated with $0,0.5,1,2,2.5,5 \mu \mathrm{M}$ melatonin for $24 \mathrm{hr}$. At the end of the incubation, aliquots were collected and centrifuged at $11,000 \times \mathrm{g}$ for 5 min at $4{ }^{\circ} \mathrm{C}$. HA in cell culture supernatants was determined according to the user manual of competitive Hyaluronan ELISA Kit (Seikagaku Corp, Tokyo, Japan). Samples were measured in triplicate and averaged. The absorbance at $450 \mathrm{~nm}$ was detected using a microplate (BIO-TEK, EL311SX).

\section{Quantitative real-time PCR}

Chondrocytes were treated with $0,0.5,1,2,2.5,5 \mu \mathrm{M}$ melatonin for $24 \mathrm{hr}$ and then collected to isolate total RNA. Method for real-time PCR has been described in our previous studies[19]. $\beta$-actin was used as the housekeeping gene. The $2^{-\Delta \Delta C t}$ method was used to assess the relative mRNA expression level. The primers used were as follows: $\quad C O X-2, \quad$ 5'-CAAACTGCTCCTGAAACCCACTC-3', 5'-GCTATTGACGATGTTCCAGACTCC-3'; Hasl, 5'-GCT CTATGGGGCGTTCCTC-3'， 5'-CACACATAAGTGGCAGGGTCC-3'; Has2 5'- 
TCGGAACCACACTGTTTGGAGTG-3',

CCAGATGTAAGTGACTGATTTGTCCC-3', Has3， 5'-ACTCTGCATCGCTGC

CTACC-3', 5'-ACATGACTTCACGCTTGCCC-3';

GAPDH,

5'-TGATGCTGGTGCTGAGTATG-3', 5'-GGTGGAAGAATGGGAGTTGC-3'.

\section{Western blot analysis}

Chondrocytes were seeded in a 6-well plate at a density of $10^{6}$ cells/well, then treated with $1 \mu \mathrm{M}$ melatonin for different periods $(0,3,6,12,24,48 \mathrm{hr})$, or treated with $0,0.5$, $1,2,2.5,5 \mu \mathrm{M}$ melatonin for $24 \mathrm{hr}$. Details of Western blot procedures can be found in our previous report[19]. Nuclear-cytoplasmic fractionation was conducted using NE - PER ${ }^{\mathrm{TM}}$ Nuclear and Cytoplasmic Extraction Reagents (Life Technologies, 78833) according to the manufacturer's instructions. Antibodies were all from Cell Signaling Technology (CST-US subsidiary, Shanghai, China) and used with a dilution of 1:1000.

\section{Immunofluorescence}

Cells were plated in six-well glass plates overnight and then washed three times with PBS before fixation, followed by permeation. Cells were then blocked and incubated overnight at $4{ }^{\circ} \mathrm{C}$ with primary antibodies. The next day, the glass plates were washed and incubated with Alexa Fluor ${ }^{\circledR}$ 488-conjugated secondary antibodies (ThermoFisher, A-11008) and labeled with DAPI for $5 \mathrm{~min}$.

\section{Mitochondrial oxygen consumption rate (OCR)}

Cells were cultured in unbuffered Dulbecco's modified Eagle medium (DMEM) at $\mathrm{pH}$ 7.4, and then transferred to wells of an XF24 Seahorse assay plate at a density of $2 \times$ $10^{5}$ cells/well to measure the mitochondrial OCR by XF analysis (XF24, Seahorse Bioscience, Billerica, MA).

\section{Cell viability assay}


Chondrocytes were seeded in a 96-well plate at a density of $10^{4}$ cells/well, then treated with $0,0.5,1,2,2.5,5 \mu \mathrm{M}$ melatonin for $24 \mathrm{hr}$. Cell viability was determined by MTS assay. The absorbance was measured using a model ELX800 Micro Plate Reader (Bio-Tek Instruments, USA) at $490 \mathrm{~nm}$.

\section{Apoptosis assay}

Cell apoptosis was estimated by flow cytometry with Annexin V-FITC /PI staining kit (Keygen Biotech, China) according to the manufacturer's instructions.

\section{Detection of mitochondrial transmembrane potential}

In brief, chondrocytes after $\mathrm{H}_{2} \mathrm{O}_{2}$ treatments were incubated with $\mathrm{Rh}-123(0.1 \mu \mathrm{g} / \mathrm{ml})$ at $37^{\circ} \mathrm{C}$ for $30 \mathrm{~min}$ and analyzed by BD flow cytometer.

\section{RNA-seq (transcriptome sequencing)}

Transcriptome sequencing based on the Illumina Hiseq sequencing platform was conducted to study mRNA expression. We used the "Cluster Profiler" R package to perform GO functional annotations and KEGG pathway enrichment analysis. The threshold values were as follows: $p<0.01$, and FDR $<0.05$.

\section{Statistical analysis}

Data were shown as mean \pm SD for at least three independent experiments. Differences between groups were determined using paired two-sided Student's t-test (groups $\leq 2$ ) or two-way ANOVA (groups $\geq 3$ ). The Bonferroni correction and Tukey's Honest Significant Difference test was used for posting hoc analysis when treatments are $>3$. A value of $P<0.05$ was considered to indicate a statistically significant difference.

\section{Results}

Melatonin protects chondrocytes from $\mathrm{H}_{2} \mathrm{O}_{2}$-induced cell injury 
MTS assay showed that melatonin at the tested concentrations was generally safe for chondrocytes (Figure 1A). To determine the solo effect of melatonin on chondrocytes, we treated chondrocytes with various concentrations of melatonin $(0,0.5,1,2,2.5,5$ $\mu \mathrm{M})$ and determined the cell viability and proliferation. Our results showed that melatonin at the tested concentrations was generally safe for chondrocytes (Figure 1A). When the chondrocytes were treated with $\mathrm{H}_{2} \mathrm{O}_{2}$ to mimic oxidative stress stimulation, we found that viability of chondrocytes was decreased, but the presence of melatonin obviously induced protective effects against $\mathrm{H}_{2} \mathrm{O}_{2}$-induced cell death at a dose-dependent manner $(* P<0.05$, $* * P<0.01)$ (Figure 1B). We chose $1 \mu \mathrm{M}$ melatonin which is in the range of the physiological serum concentration of the hormone at night as the optimum dose for subsequent experiments. Meanwhile, morphological changes of apoptosis, such as cell shrinkage and rounding, were observed after $\mathrm{H}_{2} \mathrm{O}_{2}$ treatment.

Of note, melatonin alleviated the morphological alteration of chondrocytes and reduced the occurrence of cell debris under $\mathrm{H}_{2} \mathrm{O}_{2}$ treatment (Figure 1C). These findings indicate that melatonin can protect chondrocytes from $\mathrm{H}_{2} \mathrm{O}_{2}$-induced cell injury.

As shown in Figure 1D, the apoptosis rate was increased by $\mathrm{H}_{2} \mathrm{O}_{2}(39.00 \pm 7.29 \%)$ compared to the control treatment $(2.99 \pm 3.41 \%)$. Pretreatment of chondrocytes with $1 \mu \mathrm{M}$ melatonin before $\mathrm{H}_{2} \mathrm{O}_{2}$ exposure significantly reduced the proportion of apoptotic cells compared with cultures exposed to $\mathrm{H}_{2} \mathrm{O}_{2}$ alone $(* * P<0.01)$ (Figure 1 E). The amount of cleaved PARP-1, a marker of cell apoptosis, were consistently decreased in melatonin-treated cells (Figure 1F). Together, these results suggest that melatonin protects chondrocytes from $\mathrm{H}_{2} \mathrm{O}_{2}$-induced cell injury.

\section{Melatonin upregulates HAS1 expression and HA production in chondrocytes}

To further explore the protective mechanism of melatonin on chondrocytes, we applied RNA-seq to define networks of genes regulated by melatonin. As shown in Figure 2A, after exposure to melatonin, 2596 differently expressed genes were identified in chondrocytes compared with the control group. Of the top 40 
significantly changed genes, we found 13 genes were downregulated, and 17 genes were upregulated. Among 13 downregulated genes, COX2, natriuretic peptide receptor 3 (NPR3), neural precursor cell expressed, developmentally downregulated 9 (NEDD9) related to inflammation and apoptosis, and amid 17 upregulated genes, HAS1, nicotinamide phosphoribosyltransferase (NAMPT), interferon alpha inducible protein 27 (IFI27), and were involved in OA, metabolism, and inflammation (Figure 2B). We notified that melatonin treatment upregulated HAS1 expression commonly in three independent groups, and all three members of HAS family gene were analyzed by qRT-PCR in the melatonin-treated chondrocytes. Consistent with the RNA-seq results, only HAS1 mRNA expression increased significantly after melatonin treatment $(* * P<0.01)$ (Figure 2C). Meanwhile, when chondrocytes were treated with $1 \mu \mathrm{M}$ melatonin for $0,3,6,12,24,48 \mathrm{hr}$, or treated with $0,0.5,1,2,2.5,5 \mu \mathrm{M}$ for 24 hr, the HAS1 expression was upregulated in time- and dose-dependent manners, respectively (Figure 2D). As HAS1 is the key synthesis enzyme of HA, we also detected the concentrations of HA in the culture medium of chondrocytes treated with melatonin using ELISA assay. As showed in Figure 2E, melatonin treatment resulted in a significant increase of HA concentration in cell culture supernatants under $1 \mu \mathrm{M}$ melatonin treatment over $12 \mathrm{hr}$. The level of HA in the group of $1 \mu \mathrm{M}$ melatonin increased by more than 6-fold compared with the control group (Figure 2F). The amount of HA in the medium showed a time and dose-dependent increase in the experimental groups $(* P<0.05, * * P<0.01)$ (Figure 2E, 2F). Collectively, these data showed that melatonin could increase the expression of HAS1 and HA production in chondrocytes.

\section{Melatonin exhibits anti-apoptosis activity through modulating the Akt/MAPK and NF- K B signaling in chondrocytes}

The differentially expressed genes were classified into 3 GO categories and 20 terms through gene ontology analysis (Figure 3A). The result of the cellular component analysis indicated that the expression of genes related to extracellular part, extracellular matrix, and cell-substrate junction significantly altered. The 
differentially expressed genes (DEGs) were compared with the KEGG database using BLASTX, and the corresponding pathways were established. KEGG analysis revealed significant enrichment pathways corresponding to the DEGs, among which the most significant pathways were NF- $\mathrm{B}$ signaling pathway, MAPK pathway, PI3K-Akt signaling pathway, and mitochondrial membrane and matrix part (Figure 3B). These annotations provided valuable information to investigate melatonin's effect on specific processes, functions, and pathways of chondrocytes. We further investigated whether PI3K/Akt/MAPK and NF- $\mathrm{KB}$ signaling pathways were affected by melatonin treatment. As shown in Figure 3C, the activation of Akt and ERK was increased in a concentration-dependent manner after treatment with $0.5,1,2,2.5,5 \mu \mathrm{M}$ melatonin. In contrast, melatonin resulted in reduced p65 expression. As shown in Figure 3D, melatonin obviously suppressed the p65 nuclear translocation compared with the control group. To further investigate the underlying mechanism, we detected the subcellular localization of p-p65 in $\mathrm{H}_{2} \mathrm{O}_{2}$ and melatonin treated chondrocytes. The nuclear and cytoplasmic proteins were extracted separately, and western blot analysis showed that melatonin led to accumulation of p-p65 in the cytoplasm (Figure 3E). $\mathrm{H}_{2} \mathrm{O}_{2}$ activated NF- $\kappa \mathrm{B}$ pathway, upregulated p-p65, p-IKK $\alpha / \beta$ and $\mathrm{p}-\mathrm{I} \kappa \mathrm{B} \alpha$ significantly, which was diminished in melatonin treated cells (Figure 3F), consistently with the p65 nuclear translocation observed by confocal microscopy. Altogether, these results demonstrated that melatonin protects chondrocytes from $\mathrm{H}_{2} \mathrm{O}_{2}$ induced cell injury by inhibiting the NF- $\kappa \mathrm{B}$ pathway and activating the PI3K/Akt/MAPK signaling pathway.

\section{Melatonin regulates $C O X-2$ expression and mitochondria pathway to protect chondrocytes}

We investigated the role of melatonin in the regulation of mitochondria function and found that $\mathrm{H}_{2} \mathrm{O}_{2}$ treatment significantly decreased mitochondrial membrane potential (MMP) of chondrocytes, indicating the destruction of mitochondrial integrity (Figure 4A). Cellular MMP was relatively higher in melatonin-treated cells than in cells only 
exposed to $\mathrm{H}_{2} \mathrm{O}_{2}(* * P<0.01)$ (Figure 4B, 4C). Collectively, melatonin can attenuate mitochondrial damage induced by $\mathrm{H}_{2} \mathrm{O}_{2}$. To assess which specific elements of the electron transport chain were affected by melatonin, chondrocytes were analyzed with a Seahorse Mito Stress test, and data were reported as pMoles/mg protein/min (Figure 4D). The inhibitory effect of $\mathrm{H}_{2} \mathrm{O}_{2}$ on the mitochondrial OCR was reversed by melatonin, since treatment with $1 \mu \mathrm{M}$ melatonin almost completely rescued the suppressed OCR level by $\mathrm{H}_{2} \mathrm{O}_{2}(* * P<0.01)$ (Figure 4E). As melatonin is widely known for its antioxidant and anti-inflammatory properties, we subsequently examined the expression of inflammation-associated molecules. COX-2 is frequently upregulated in OA joints and may further affect HA synthesis ${ }^{11}$. Therefore, we detected the effect of melatonin on the expression of COX-2 mRNA. As showed in Figure 4F, treatment of melatonin effectively decreased the expression of $C O X-2$ mRNA in chondrocytes in a dose-dependent manner $(* P<0.05$, $* * P<0.01)$. As COX-2 is a well-known NF- $\mathrm{KB}$ target gene, we next examined melatonin's effect on the expression of COX-2 and NF- $\kappa$ B family members. As showed in Figure 4G, $1 \mu \mathrm{M}$ could effectively decreased the expression of COX-2, p-p65, p-IKK $\alpha / \beta$ and p-IкB $\alpha$ levels. Taken together, our data indicate that melatonin repressed COX-2 expression and the canonical NF-кB pathway activation. Therefore, melatonin may mitigate the expression of inflammatory molecules and restore mitochondrial function under oxidative stress in chondrocytes.

\section{Discussion}

In this study, we identified melatonin as an anti-inflammatory and antioxidant agent of chondrocytes under oxidative stress. Melatonin could repress NF- $\kappa \mathrm{B}$ signaling and attenuate the expression of inflammation-associated molecule COX-2, maintain HA production, and chondrocytes survival against oxidative stress.

Melatonin levels vary throughout human life and are known to decline with age. It is assumed that melatonin downregulation in serum is a consequence of aging, which contributes to the generation of aging-associated diseases[20]. Emerging evidence has 
also indicated that melatonin is associated with bone remodeling, osteoporosis, and osseointegration of dental implants[21]. Melatonin modulates bone formation and osteoblast differentiation of bone marrow mesenchymal cells[22]. Previous studies show that melatonin provides protective effects for cartilage destruction caused by inflammatory arthritis[23]. Melatonin reduces TNF- $\alpha$ and IL-1 $\beta$ production in synovial fibroblasts and diminishes cartilage degradation[23]. In addition, melatonin is shown to stimulate cartilage repairment through modulating the expression of the circadian clock genes, such as BMAL, CRY, and DEC2[24]. In an OA model, intra-articular injection of melatonin effectively attenuated cartilage degradation[25]. Melatonin can enhance cartilage matrix synthesis by porcine articular chondrocytes[26]. Melatonin inhibits the excessive proliferation and migration of human fibroblast-like synoviocytes[27]. Taken together, melatonin might be a promising therapeutic agent for OA treatment due to its multifaceted functions, such as alleviating inflammation, maintaining metabolic balance, and mitigating apoptosis[28].

Studies have shown that ROS contributes to the onset and progression of OA, giving rise to chondrocyte hypertrophy, apoptosis, extracellular matrix synthesis, and degradation as well as synovial inflammation and dysfunction of the subchondral bone[6]. In our study, chondrocytes were exposed to $400 \mu \mathrm{M} \mathrm{H}_{2} \mathrm{O}_{2}$ to induce cell apoptosis. We observed that melatonin ameliorated $\mathrm{H}_{2} \mathrm{O}_{2}$-induced cell apoptosis and mitochondrial damage. Therefore, our finding validates that melatonin facilitates chondrocytes to tolerate oxidative stress. Meanwhile, melatonin mitigated $\mathrm{H}_{2} \mathrm{O}_{2}$-induced expression of inflammatory molecules, as evidenced by reduced $C O X-2$ expression and inhibited NF- $\kappa \mathrm{B}$ signal. NF- $\kappa \mathrm{B}$ signal alters the expression of various genes involved in cartilage destruction, synovial membrane inflammation, and subchondral bone resorption. Therefore, it has long been recognized as a disease-contributing factor and has become a therapeutic target for OA[29, 30].In this study, we found that melatonin decreased the expression of the NF- $\mathrm{B}$ family members p50 and p65. COX-2 expression is induced in inflammatory conditions due 
to different inflammatory stimuli including cytokines. COX-2 has an important regulatory function in promoting chondrocyte hypertrophic differentiation[31]. Thus, melatonin may inhibit $\mathrm{NF}-\kappa \mathrm{B}$ signal to affect COX-2 expression and chondrocyte function. Collectively, our results showed that melatonin could antagonize oxidative stress and inflammation to protect chondrocytes.

In addition, we provide evidence that melatonin promoted HA production in concentration- and time-dependent manners. HA plays a crucial role in the lubricating, buffering functions during joint motion and inhibiting the production of inflammatory molecules to mitigate cartilage degeneration[32]. In addition, HA even can reduce pain sensation by decreasing COX-2 levels[33]. In OA joints, inflammation decreases the production of HA from chondrocytes and synoviocytes. HA concentration and its molecular weight in injured joints of OA patients are lower than those in healthy people[34]. The amount of HA and its molecular weight distribution is decided by the balance between its synthesis and degradation. HA is synthesized into the extracellular space by hyaluronan synthase (HAS)[35]. Three distinct HAS isoforms (HAS1, HAS2, and HAS3) are identified in mammalian cells, differing in catalytic properties and distribution[36]. HAS2 is predominantly expressed in normal cells, while HAS3 is mainly expressed in cancer cells[37]. HAS2 is the most abundant isozyme expressing in particular chondrocytes and yields high molecular weight HA contributing to the maintenance of the cartilage matrix [38]. Taken together, melatonin might be capable of maintaining the production and qualification of HA in joints as well as counteracting the inhibitory effects of oxidative stress.

\section{Conclusion}

Overall, our study shows that melatonin exerts a protective effect on chondrocytes. Melatonin enhances HA synthesis, attenuates oxidative injury, and inflammation. Combined with previous studies, our finding strongly implicates that melatonin protects the articular cartilage from OA-induced damage. Melatonin supplement may represent alternative prevention and treatment for OA. 
Authors' Contributions: JJ.W., PY.H., Y.X., X. L., and HM.J. performed the experiments; YX.W., S.W., and J.G. analyzed the data; ZQ.L. and JY.W. designed the research study, and A.T., JJ.W., and ZQ.L. prepared the manuscript.

Declaration of Conflicting Interests: The authors declared no potential conflicts of interest with respect to the research, authorship, and publication of this article.

Funding: This work was supported by the Talent Project of Tianjin Medical University (11601501/2016KJ0317), the College Student Innovation Program of Tianjin Medical University (202010062030, ZY.P.), and the Tianjin Research Innovation Project for Postgraduate Students (2019YJSB110, JJ.W.; 2020YJSB162, Y.X.). 


\section{References}

[1] V. Santilli, M. Paoloni, M. Mangone, F. Alviti, A. Bernetti, Hyaluronic acid in the management of osteoarthritis: injection therapies innovations, Clinical cases in mineral and bone metabolism : the official journal of the Italian Society of Osteoporosis, Mineral Metabolism, and Skeletal Diseases, 13 (2016) 131-134.

[2] M.H. Ebell, Osteoarthritis: Rapid Evidence Review, American family physician, 97 (2018) 523-526.

[3] M.B. Goldring, K.B. Marcu, Cartilage homeostasis in health and rheumatic diseases, Arthritis research \& therapy, 11 (2009) 224.

[4] T. Mao, C. He, H. Wu, B. Yang, X. Li, Silencing IncRNA HOTAIR declines synovial inflammation and synoviocyte proliferation and promotes synoviocyte apoptosis in osteoarthritis rats by inhibiting Wnt/beta-catenin signaling pathway, Cell Cycle, 18 (2019) 3189-3205.

[5] H.S. Hwang, H.A. Kim, Chondrocyte Apoptosis in the Pathogenesis of Osteoarthritis, Int J Mol Sci, 16 (2015) 26035-26054.

[6] P. Lepetsos, A.G. Papavassiliou, ROS/oxidative stress signaling in osteoarthritis, Biochimica et biophysica acta, 1862 (2016) 576-591.

[7] B. Hoesel, J.A. Schmid, The complexity of NF-kappaB signaling in inflammation and cancer, Mol Cancer, 12 (2013) 86.

[8] G. Bonizzi, M. Karin, The two NF-kappaB activation pathways and their role in innate and adaptive immunity, Trends Immunol, 25 (2004) 280-288.

[9] S.C. Sun, The non-canonical NF-kappaB pathway in immunity and inflammation, Nat Rev Immunol, 17 (2017) 545-558.

[10] M.S. Hayden, S. Ghosh, Shared principles in NF-kappaB signaling, Cell, 132 (2008) 344-362.

[11] K.B. Marcu, M. Otero, E. Olivotto, R.M. Borzi, M.B. Goldring, NF-kappaB signaling: multiple angles to target OA, Curr Drug Targets, 11 (2010) 599-613.

[12] A. Sfikas, C. Batsi, E. Tselikou, G. Vartholomatos, N. Monokrousos, P. Pappas, S. Christoforidis, T. Tzavaras, P. Kanavaros, V.G. Gorgoulis, K.B. Marcu, E. Kolettas, The canonical NF-kappaB pathway differentially protects normal and human tumor cells from ROS-induced DNA damage, Cell Signal, 24 (2012) 2007-2023.

[13] B. Bartok, G.S. Firestein, Fibroblast-like synoviocytes: key effector cells in rheumatoid arthritis, Immunol Rev, 233 (2010) 233-255.

[14] M.B. Goldring, M. Otero, Inflammation in osteoarthritis, Curr Opin Rheumatol, 23 (2011) 471-478.

[15] P. Singh, K.B. Marcu, M.B. Goldring, M. Otero, Phenotypic instability of chondrocytes in osteoarthritis: on a path to hypertrophy, Annals of the New York Academy of Sciences, 1442 (2019) 17-34.

[16] B. Claustrat, J. Leston, Melatonin: Physiological effects in humans, Neurochirurgie, 61 (2015) 77-84.

[17] S. Tordjman, S. Chokron, R. Delorme, A. Charrier, E. Bellissant, N. Jaafari, C. Fougerou, Melatonin: Pharmacology, Functions and Therapeutic Benefits, Current neuropharmacology, 15 (2017) 434-443.

[18] G. Favero, L. Franceschetti, F. Bonomini, L.F. Rodella, R. Rezzani, Melatonin as an Anti-Inflammatory Agent Modulating Inflammasome Activation, International journal of endocrinology, 2017 (2017) 1835195.

[19] J. Liu, Y. Xie, J. Guo, X. Li, J. Wang, H. Jiang, Z. Peng, J. Wang, S. Wang, Q. Li, L. Ye, Y. Zhong, Q. Zhang, X. Liu, D.M. Lonard, J. Wang, B.W. O'Malley, Z. Liu, Targeting NSD2-mediated SRC-3 liquid-liquid phase separation sensitizes bortezomib treatment in multiple myeloma, Nat Commun, 12 (2021) 1022. 
[20] M. Majidinia, R.J. Reiter, S.K. Shakouri, I. Mohebbi, M. Rastegar, M. Kaviani, S.G. Darband, R. Jahanban-Esfahlan, S.M. Nabavi, B. Yousefi, The multiple functions of melatonin in regenerative medicine, Ageing research reviews, 45 (2018) 33-52.

[21] J. Liu, F. Huang, H.W. He, Melatonin effects on hard tissues: bone and tooth, International journal of molecular sciences, 14 (2013) 10063-10074.

[22] M. Liu, J. Ai, J. Feng, J. Zheng, K. Tang, Z. Shuai, J. Yang, Effect of paeoniflorin on cardiac remodeling in chronic heart failure rats through the transforming growth factor beta1/Smad signaling pathway, Cardiovascular diagnosis and therapy, 9 (2019) 272-280.

[23] C.C. Huang, C.H. Chiou, S.C. Liu, S.L. Hu, C.M. Su, C.H. Tsai, C.H. Tang, Melatonin attenuates TNF-alpha and IL-1beta expression in synovial fibroblasts and diminishes cartilage degradation: Implications for the treatment of rheumatoid arthritis, J Pineal Res, 66 (2019) e12560.

[24] R. Jahanban-Esfahlan, S. Mehrzadi, R.J. Reiter, K. Seidi, M. Majidinia, H.B. Baghi, N. Khatami, B. Yousefi, A. Sadeghpour, Melatonin in regulation of inflammatory pathways in rheumatoid arthritis and osteoarthritis: involvement of circadian clock genes, British journal of pharmacology, 175 (2018) 3230-3238.

[25] H.D. Lim, Y.S. Kim, S.H. Ko, I.J. Yoon, S.G. Cho, Y.H. Chun, B.J. Choi, E.C. Kim, Cytoprotective and anti-inflammatory effects of melatonin in hydrogen peroxide-stimulated $\mathrm{CHON}-001$ human chondrocyte cell line and rabbit model of osteoarthritis via the SIRT1 pathway, Journal of pineal research, 53 (2012) 225-237.

[26] M. Pei, F. He, L. Wei, A. Rawson, Melatonin enhances cartilage matrix synthesis by porcine articular chondrocytes, Journal of pineal research, 46 (2009) 181-187.

[27] S.S. Nah, H.J. Won, H.J. Park, E. Ha, J.H. Chung, H.Y. Cho, H.H. Baik, Melatonin inhibits human fibroblast-like synoviocyte proliferation via extracellular signal-regulated protein kinase/P21(CIP1)/P27(KIP1) pathways, Journal of pineal research, 47 (2009) 70-74.

[28] F.M. Hossain, Y. Hong, Y. Jin, J. Choi, Y. Hong, Physiological and Pathological Role of Circadian Hormones in Osteoarthritis: Dose-Dependent or Time-Dependent?, Journal of clinical medicine, 8 (2019).

[29] M.C. Choi, J. Jo, J. Park, H.K. Kang, Y. Park, NF-kappaB Signaling Pathways in Osteoarthritic Cartilage Destruction, Cells, 8 (2019).

[30] S. Rigoglou, A.G. Papavassiliou, The NF-kappaB signalling pathway in osteoarthritis, Int J Biochem Cell Biol, 45 (2013) 2580-2584.

[31] T.J. Welting, M.M. Caron, P.J. Emans, M.P. Janssen, K. Sanen, M.M. Coolsen, L. Voss, D.A. Surtel, A. Cremers, J.W. Voncken, L.W. van Rhijn, Inhibition of cyclooxygenase-2 impacts chondrocyte hypertrophic differentiation during endochondral ossification, European cells \& materials, 22 (2011) 420-436; discussion 436-427.

[32] C.T. Wang, Y.T. Lin, B.L. Chiang, Y.H. Lin, S.M. Hou, High molecular weight hyaluronic acid down-regulates the gene expression of osteoarthritis-associated cytokines and enzymes in fibroblast-like synoviocytes from patients with early osteoarthritis, Osteoarthritis and cartilage, 14 (2006) 1237-1247.

[33] Y. Mitsui, M. Gotoh, K. Nakama, T. Yamada, F. Higuchi, K. Nagata, Hyaluronic acid inhibits mRNA expression of proinflammatory cytokines and cyclooxygenase-2/prostaglandin $E(2)$ production via CD44 in interleukin-1-stimulated subacromial synovial fibroblasts from patients with rotator cuff disease, Journal of orthopaedic research : official publication of the Orthopaedic Research Society, 26 (2008) 1032-1037. 
[34] L.B. Dahl, I.M. Dahl, A. Engstrom-Laurent, K. Granath, Concentration and molecular weight of sodium hyaluronate in synovial fluid from patients with rheumatoid arthritis and other arthropathies, Annals of the rheumatic diseases, 44 (1985) 817-822.

[35] P.H. Weigel, Hyaluronan Synthase: The Mechanism of Initiation at the Reducing End and a Pendulum Model for Polysaccharide Translocation to the Cell Exterior, Int J Cell Biol, 2015 (2015) 367579.

[36] N. Itano, K. Kimata, Mammalian hyaluronan synthases, IUBMB Life, 54 (2002) 195-199.

[37] R.H. Tammi, A.G. Passi, K. Rilla, E. Karousou, D. Vigetti, K. Makkonen, M.I. Tammi, Transcriptional and post-translational regulation of hyaluronan synthesis, The FEBS journal, 278 (2011) 1419-1428.

[38] D.R. Hiscock, B. Caterson, C.R. Flannery, Expression of hyaluronan synthases in articular cartilage, Osteoarthritis and cartilage, 8 (2000) 120-126. 


\section{Figure legends}

A

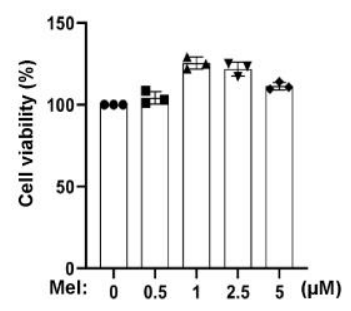

B

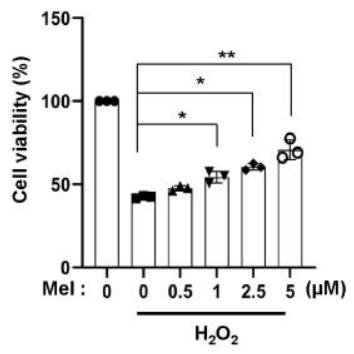

C

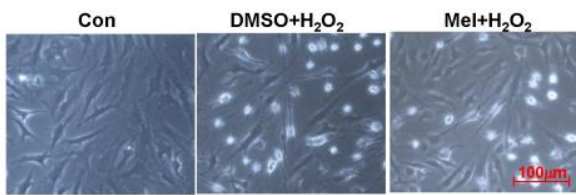

D

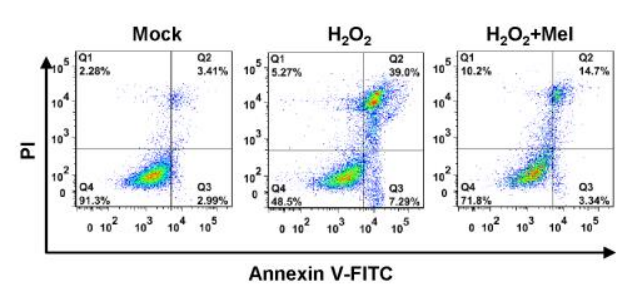

E

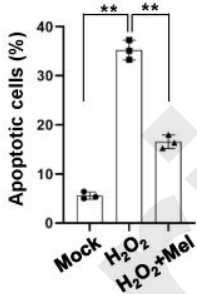

$\mathbf{F}$

\section{Figure 1. Melatonin protects chondrocytes from $\mathrm{H}_{2} \mathrm{O}_{2}$ induced injury.}

(A) Chondrocytes viability was estimated by MTS assay after incubated with increasing concentrations of melatonin for $24 \mathrm{hr}$. DMSO was used as a control.

Chondrocytes were pretreated with melatonin for $24 \mathrm{hr}$ and then exposed to $400 \mu \mathrm{M}$ $\mathrm{H}_{2} \mathrm{O}_{2}$ in the presence of melatonin. MTS assay was performed to evaluate the cell viability. (C) Morphological changes of chondrocytes after $400 \mu \mathrm{M} \mathrm{H}_{2} \mathrm{O}_{2}$ treatment in the presence or absence of $1 \mu \mathrm{M}$ melatonin. (D) The apoptosis of chondrocytes was assessed by Annexin V-FITC/PI double staining after treatment; one typical images of each group is shown. (E) The apoptotic rates are quantified by Graphpad Prism 8. (F) Western blots show the presence of cleaved PARP-1 in chondrocytes after $\mathrm{H}_{2} \mathrm{O}_{2}$ and melatonin treatment for $24 \mathrm{hr}$. Data obtained from three independent experiments are shown as mean $\pm \mathrm{SD} .{ }^{*} P<0.05, * * P<0.01$ compared with control. 
A

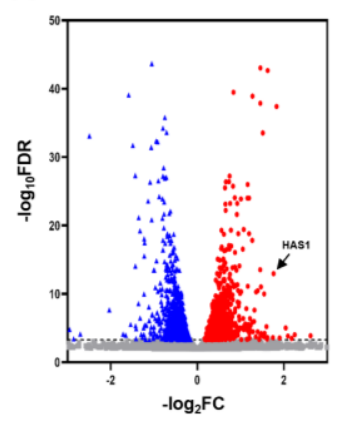

D

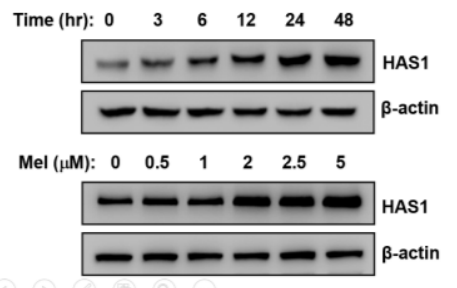

B

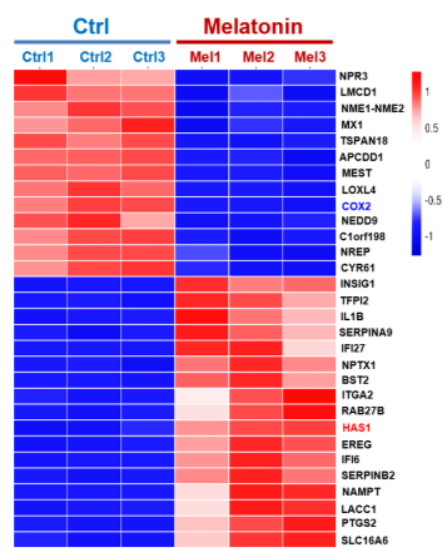

E
C

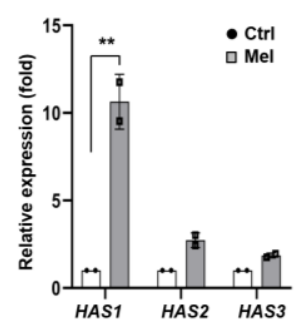

Figure 2. RNA-seq screened HAS1 as the main target gene of melatonin in chondrocyte.

(A) Volcano plot displayed the differentially expressed genes between melatonin and control groups. The vertical gray lines corresponded to two-fold up- and down-regulation ( $\log 2$ scaled), and the horizontal gray line represented a $p$-value of 0.05. (B) Heatmap of top 30 different expression genes (independent group $t$-test $P<0.05$ and fold difference $\geqslant 2$ ). (C) The relative expression levels of HAS family gene. (D) Chondrocytes were treated with $1 \mu \mathrm{M}$ melatonin for increasing time or increasing concentrations of melatonin for $24 \mathrm{hr}$, and HAS1 was detected with western blot. (E) After treated with $1 \mu \mathrm{M}$ melatonin for various times, HA production in the chondrocytes culture supernatants was measured by ELISA. (F) After $24 \mathrm{hr}$ treated with melatonin for various doses, HA production in the cell culture supernatants was measured by ELISA. Data obtained from three independent experiments were shown as mean $\pm \mathrm{SD} . * P<0.05, * * P<0.01$ compared with control. 

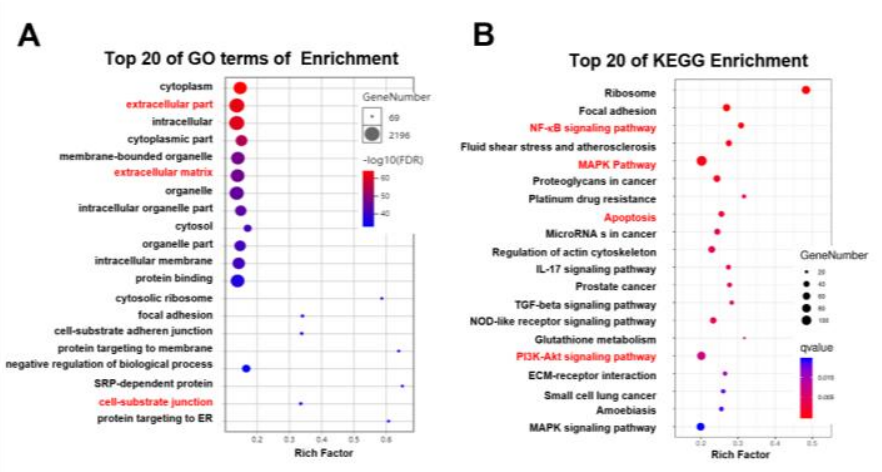

C
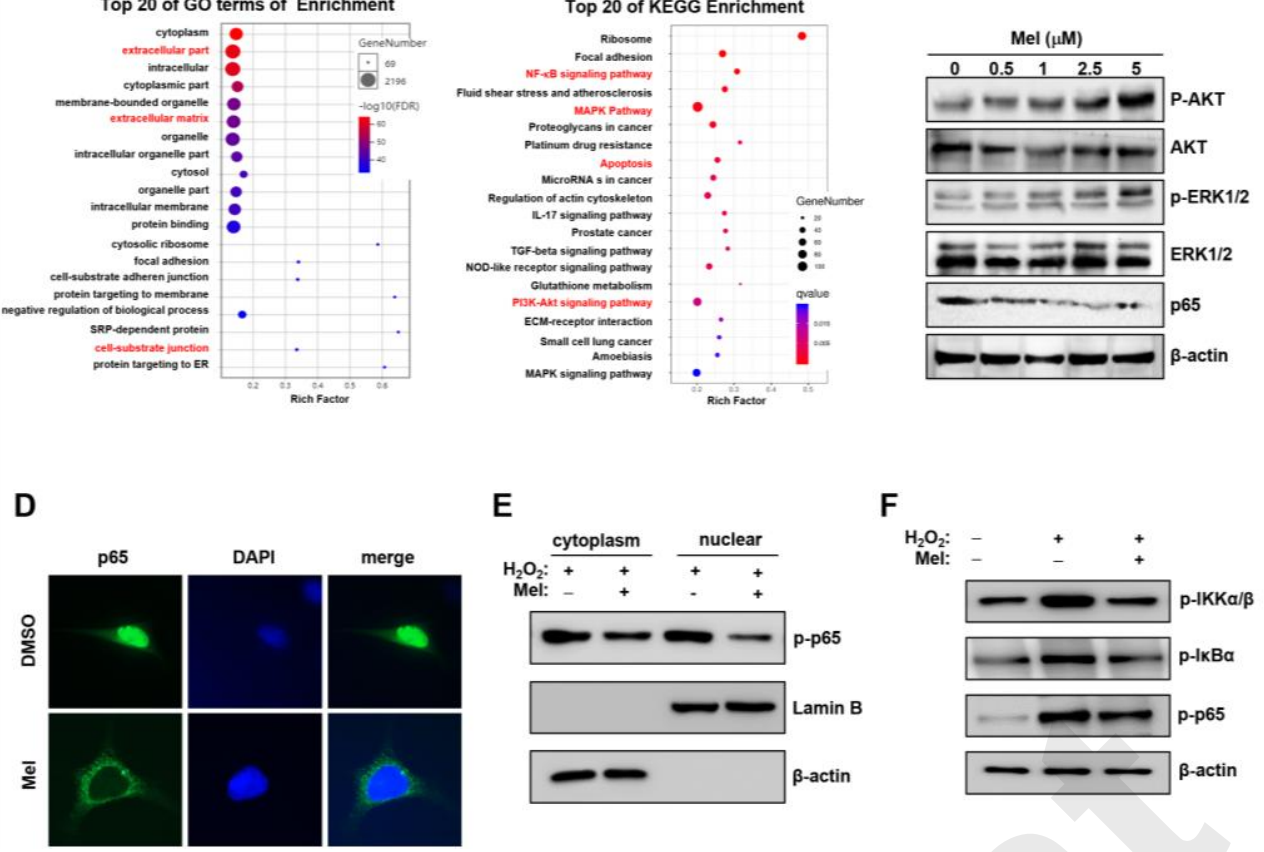

Figure 3. KEGG or GO analysis revealed the activation of AKT and MAPK signaling, inhibiting NF-kB.

(A) GO annotations of the differentially expressed genes. (B) Bulb map of KEGG analysis for the host genes of differentially expressed genes. The statistical significance increased from purple (relatively lower significance) to red (relatively higher significance). (C) Western blot showed PI3K/Akt/MAPK signaling pathway. Mark genes expression after melatonin treatment. (D) p65 location was determined using immunofluorescence confocal microscopy, and DAPI was used to localize cell nuclei. Scale bar, $10 \mu \mathrm{m}$. (E) Protein levels of p-p65 in the cytoplasmic and nuclear fractions were measured by western blot after $\mathrm{H}_{2} \mathrm{O}_{2}$ and melatonin treatment for $24 \mathrm{hr}$. Lamin B was used as nuclear protein control. (F) Western blot to show the alternation of p-p65, p-IKK $\alpha / \beta$ and p-I $\kappa \mathrm{B} \alpha$ protein levels in the WT chondrocytes after $\mathrm{H}_{2} \mathrm{O}_{2}$ and melatonin treatment for $24 \mathrm{hr}$. Data obtained from three independent experiments were shown as mean $\pm \mathrm{SD} .{ }^{*} P<0.05, * * P<0.01$ compared with control. 
A
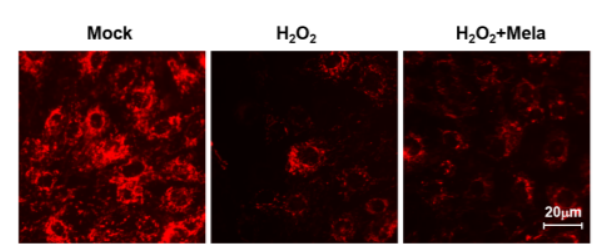

B

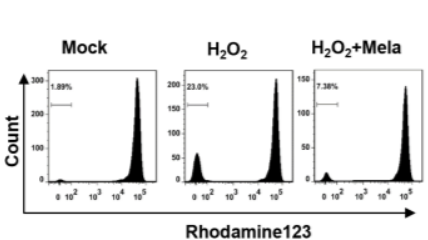

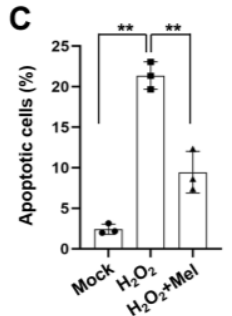

G

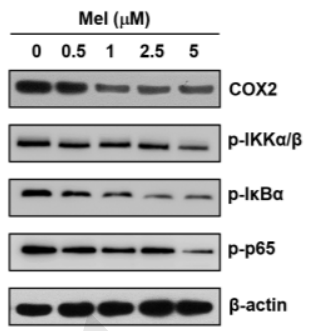

Figure 4. Melatonin alleviates cell apoptosis and mitochondrial dysfunction.

(A) Mitochondrial membrane potential was detected using Rh-123 staining, scale bar, $20 \mu \mathrm{m}$ (B) and analyzed by flow cytometry. (C) The relative fluorescence intensity is quantitated. One typical image of three similar experiments is shown. (D) Oxygen consumption rate (OCR) was determined using Seahorse XF Mito Stress analysis in chondrocytes treated by $400 \mu \mathrm{M} \mathrm{H}_{2} \mathrm{O}_{2}$ in the presence or absence of $1 \mu \mathrm{M}$ melatonin for $24 \mathrm{hr}$. (E) Respective bar graphs of maximal OCR. (F) The relative mRNA expression of $C O X-2$ under $0 \sim 5 \mu \mathrm{M}$ melatonin for $24 \mathrm{hr}$. (G) Western blot to show the decreased expression of COX-2, p-p65, p-IKK $\alpha / \beta$ and $\mathrm{p}-\mathrm{I} \kappa \mathrm{B} \alpha$ after treated with increasing concentrations of melatonin for $24 \mathrm{hr}$. Data mean $\pm \mathrm{SD}$. $* P<0.05$, $* * P<$ 0.01, analyzed by one-way ANOVA with Fisher's LSD. 
A

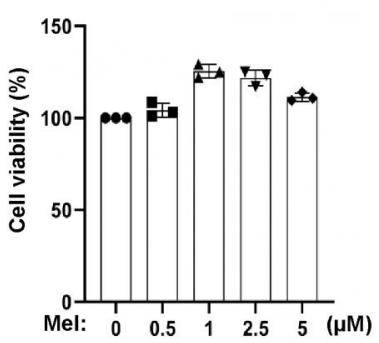

B

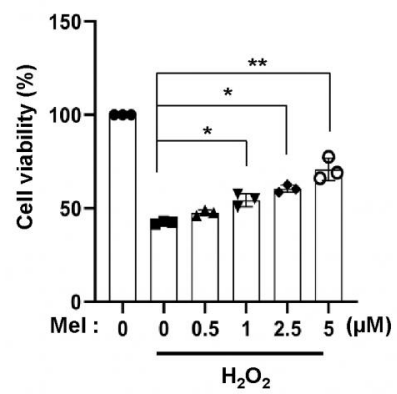

C

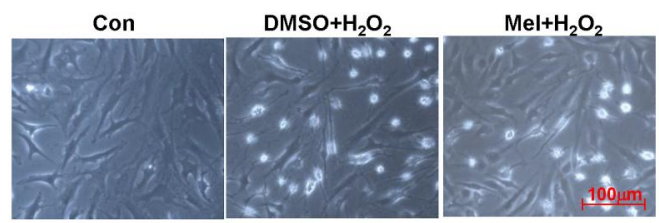

E

F

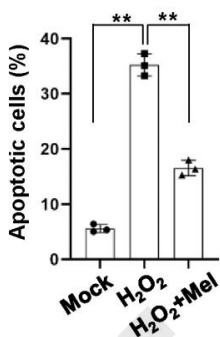

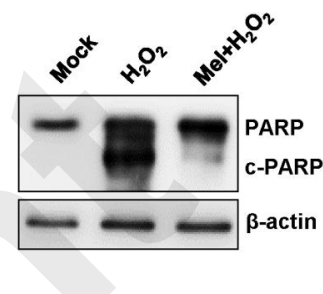




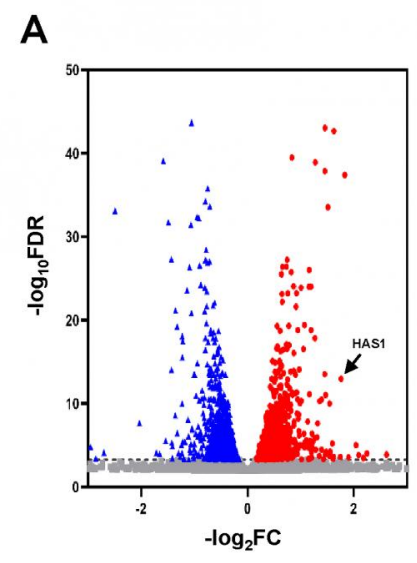

B

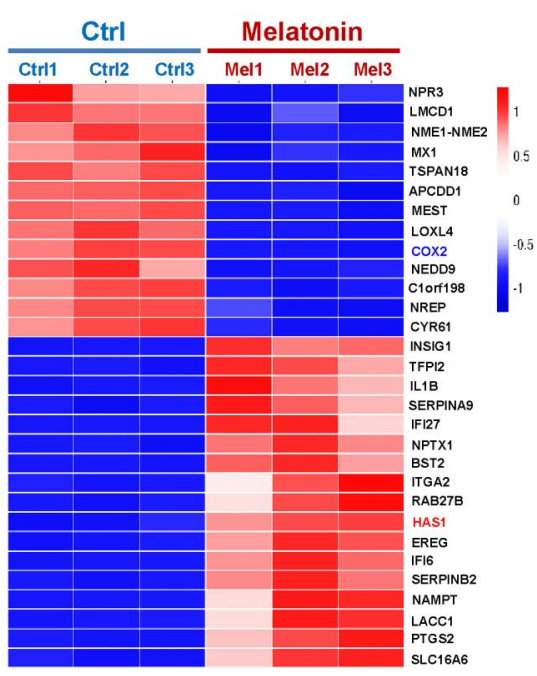

E

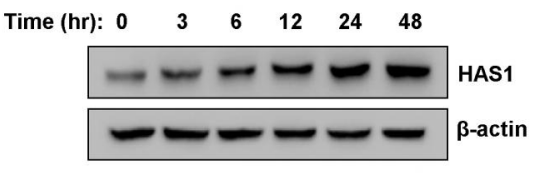

$\operatorname{Mel}(\mu \mathrm{M}): \quad \begin{array}{llllll}0 & 0.5 & 1 & 2 & 2.5 & 5\end{array}$

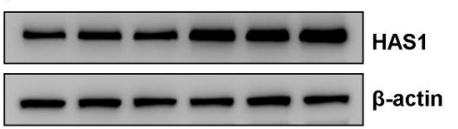

F

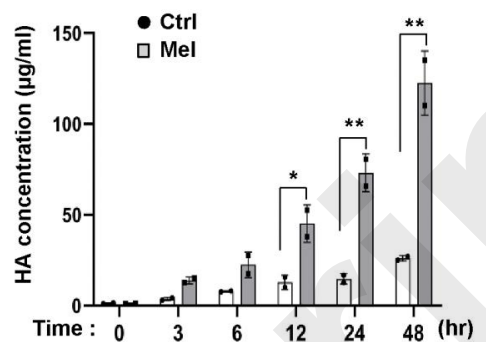

C

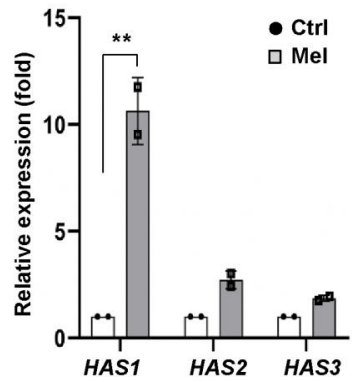

G

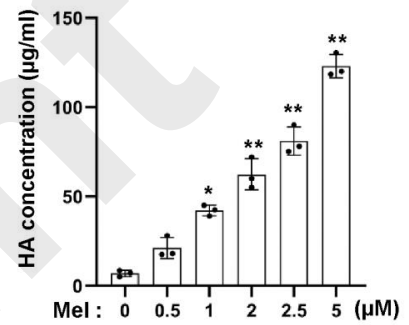


A

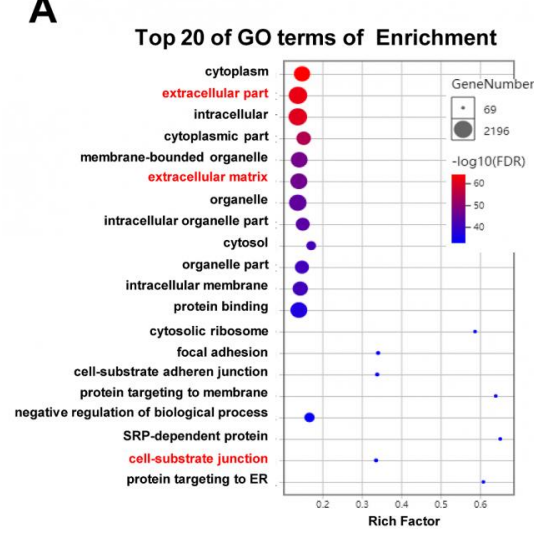

D

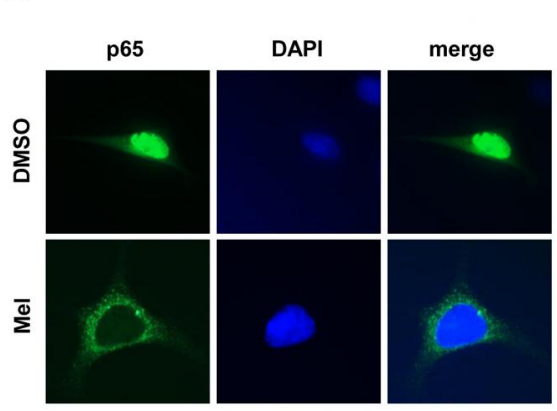

B

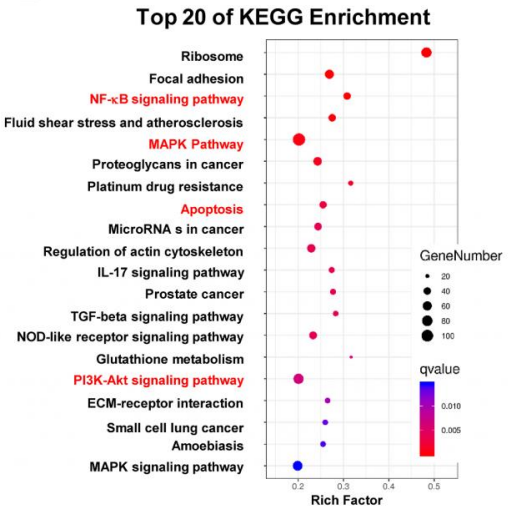

E

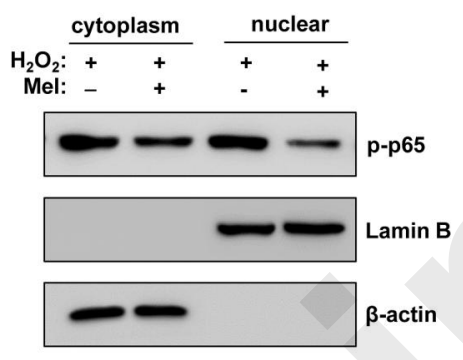

C

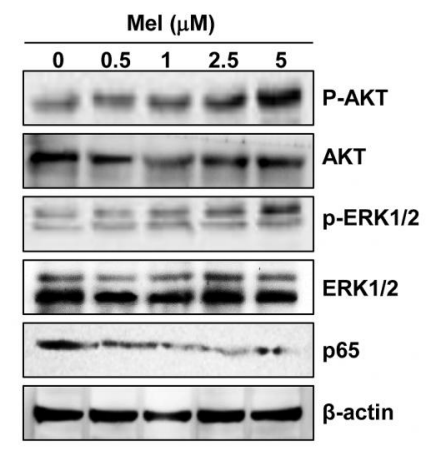

F $\mathrm{H}_{2} \mathrm{O}_{2}:$
Mel:

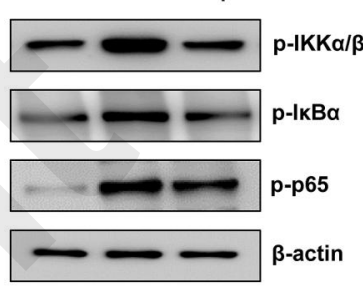


A
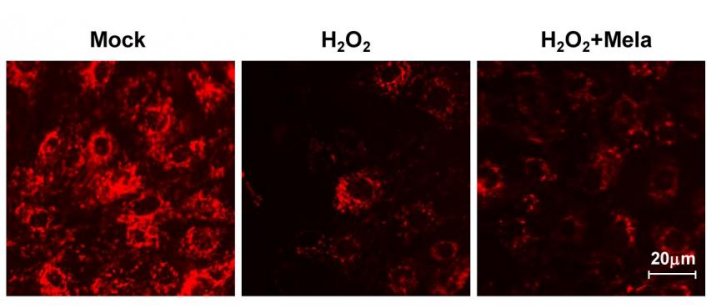

D

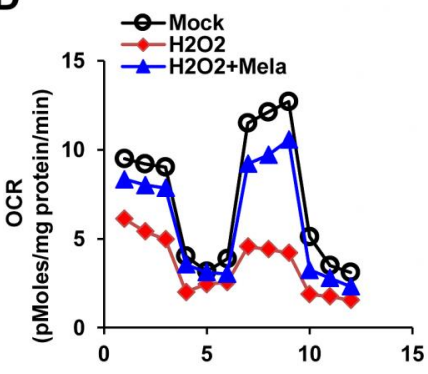

B

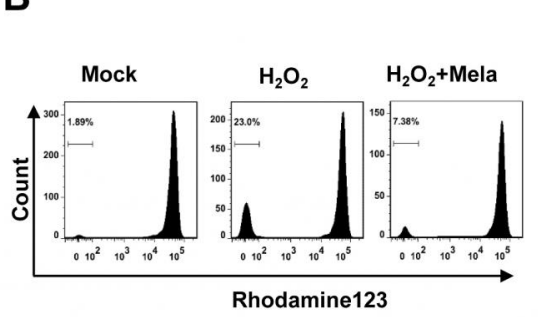

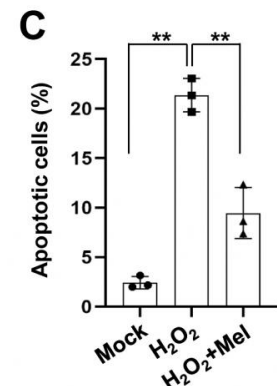

F

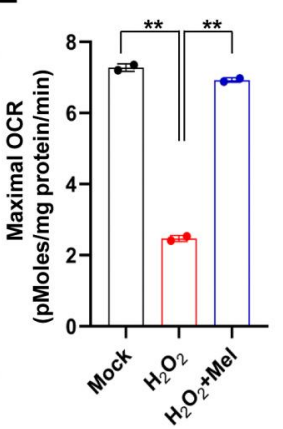

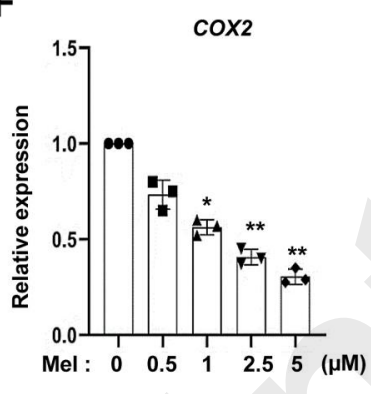

G

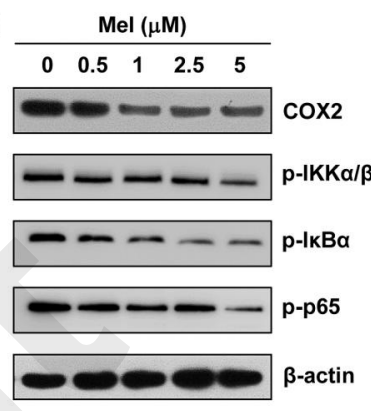

